\title{
Random spectrum fatigue of zirconia oral implants in air and saline solution
}

\author{
Shemtov-Yona, K ; Özcan, M ; Godinger, A ; de Basso, N ; Rittel, D
}

\begin{abstract}
Zirconia oral implants are increasingly considered as an alternative to metallic (titanium) ones, mostly for their aesthetic properties and their metal free composition that enables soft tissue integration. We report a systematic comparison of the fatigue performance of oral implants of identical geometry, made of partially stabilized zirconia (Y-TZP) and titanium as a reference group, using random spectrum fatigue testing in both air and saline solution. The spectrum data clearly points unequivocally to the operation of a fatigue (damage accumulation) failure mechanism in the zirconia implants. Saline solution reduces the quasi-static fracture strength. It also causes a marked degradation of their spectrum fatigue longevity, but it does not affect their spectrum fatigue fracture strength. Given that the quasi-static fracture strength of the tested implants is smaller in saline solution than in air, the results suggest that the maximum admissible design loads for spectrum loaded zirconia oral implants are of the order of 0.8 times their wet (saline) quasi-static fracture strength.
\end{abstract}

DOI: https://doi.org/10.1016/j.engfailanal.2019.104160

Posted at the Zurich Open Repository and Archive, University of Zurich

ZORA URL: https://doi.org/10.5167/uzh-183020

Journal Article

Accepted Version

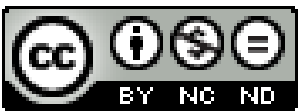

The following work is licensed under a Creative Commons: Attribution-NonCommercial-NoDerivatives 4.0 International (CC BY-NC-ND 4.0) License.

Originally published at:

Shemtov-Yona, K; Özcan, M; Godinger, A; de Basso, N; Rittel, D (2019). Random spectrum fatigue of zirconia oral implants in air and saline solution. Engineering Failure Analysis, 106:104160.

DOI: https://doi.org/10.1016/j.engfailanal.2019.104160 


\title{
Random spectrum fatigue of zirconia dental implants in air and saline solution
}

\author{
K. Shemtov-Yona1*, M. Özcan2, A. Godinger1, N. de Basso3 and D. Rittel1 \\ 1Faculty of Mechanical Engineering, Technion, 32000 Haifa, Israel \\ ${ }_{2}$ Center for Dental and Oral Medicine, University of Zurich, CH-8032, Zurich, Switzerland \\ 3 Private Practice, Stockholm, Sweden \\ Declarations of interest: none
}

\begin{abstract}
Zirconia dental implants are often considered as an alternative to metallic (titanium) ones, mostly for their aesthetic properties and their metal free composition that allow for better soft tissue integration.

We report a systematic comparison of the fatigue performance of dental implants of identical geometry, made of partially stabilized zirconia (Y-TZP) and titanium as a reference group, using random spectrum fatigue testing in both air and saline solution.

It is observed that saline solution reduces the static fracture strength of the zirconia implants. It also causes a marked degradation of their spectrum fatigue longevity, but it does not affect their spectrum fatigue fracture strength.

The results suggest that the maximum admissible design loads for spectrum loaded zirconia dental implants are of the order of 0.8 times their wet static fracture strength.
\end{abstract}

Keywords: dental implants, zirconia, titanium, random spectrum, fatigue, air, saline solution 


\section{INTRODUCTION}

Oral implants offer an effective treatment for replacement of missing teeth. Current long-term clinical investigations, with over 20 years of follow-up, report very high survival rates which place titanium and its biomedical alloys as the gold standard [1-3].

With that, modern dental applications add aesthetic requirements (color, translucency) to the mechanical/functional specifications. White to ivory color confers a clear advantage to the aesthetic outcome so that research and development are nowadays directed towards metal-free dental prosthetic restorations that preserve soft tissue color. For their similarity to the natural teeth, ceramic base materials are increasingly used to fabricate crowns, abutments and also implants $[4,5]$.

Zirconia ceramics (yttria partially stabilized tetragonal zirconia) have demonstrated excellent biocompatibility, mechanical characteristics and superior esthetic outcome. Furthermore, they can be used with patients suffering from allergies to metals [6,7]. Several animal studies have shown that the success of the osseointegration processes of ceramic dental implants is comparable to that of titanium and its alloys [8].

Among the most promising candidates, yttria partially stabilized polycrystalline zirconia (YTZP) has a metastable tetragonal structure that can undergo a stress-induced phase transformation at room temperature, resulting in the formation of the stable monoclinic structure. It has been shown that this phase transformation strengthens and toughens the YTZP, whose strength and fracture toughness may exceed those of reinforced alumina and other classes of zirconia based ceramics [4,9-12].

However, a systematic review, evaluating the clinical success and survival rates of zirconia single-body and two-body implants after at least 1 year of function, showed that the survival rate was 92\% [13]. When longer follow up times were considered, the survival rate was significantly reduced. Roehling et al. [14] investigated retrospectively the clinical performance of first-generation zirconia implants with a sandblasted surface, up to and beyond 7 years of service. The survival rate of 161 zirconia implants after 7 year was found to be only $77.6 \%$, with a total of $36(22.4 \%)$ failures. $50 \%$ of the failures were due to fracture after prosthetic loading $(n=18)$. Such a high incidence of mechanical implant fractures exceeds markedly that reported for titanium-base metallic implants, which is of the order of $4 \%$ over 5 years [15].

In order to evaluate the mechanical reliability and long term behavior of zirconia implants, invitro testing should ideally be performed under cyclic loading in a way that can simulate the implant's service conditions [16,17]. Those authors report a decrease of implant fracture strength after cyclic loading. In vitro tests can be divided into two main categories, namely the mechanical and the biochemical environment, and combinations thereof. Implants are load- 
bearing medical devices aimed to function under a complex mechanical environment of mastication loads, which are not supposed to reach the failure strength of the material. The chemical environment is extremely complex as the implants are exposed to different media with different electrolyte concentrations and $\mathrm{pH}$ [18]. The potential interaction between the mechanical loads and biochemical atmosphere may impair the long-term mechanical performance of the zirconia ceramic implant, just like stress-corrosion would do for a metallic material.

The mechanical properties of ceramics are usually characterized in terms of hardness, fracture toughness and bending strength. The use of ceramics for bio-structural applications has been partly motivated by the prospect that they may be insensitive to mechanical degradation associated with fatigue loading, a subject that has been widely investigated. As of today, the prevailing view is that ceramics can indeed experience fatigue failure [19-22], although the responsible micromechanisms are totally different than those observed in metals [23]. Crack growth rate was found to be sensitive to several parameters, such as the stress intensity range, frequency and load ratio [11,19]. It was also reported that ceramic materials undergo crack closure associated with the dilatation of the stress-transformed phase, that can be interpreted as crack-tip shielding during cyclic loading $[11,24,25]$.

It is important to mention that Dauskardt et al. [20] showed that cyclic crack growth rates can also be influenced by the cycling history under variable amplitude loading, thereby emphasizing the loading sequence in itself and not only the cyclic load amplitude. Those authors concluded that variable amplitude cyclic testing of ceramics is the optimal way to investigate their fatigue properties.

Environmental influence has been reported on mechanical properties of Y-TZP $[12,26]$. Here, the tetragonal to monoclinic phase transformation [27] acts as a double-edged sword, making Y-TZP prone to low temperature degradation (sometimes referred to as aging) in the presence of water, resulting in surface roughening (swelling) and surface micro-cracking. Aging is usually considered at "low" temperatures $\left(65-300{ }^{\circ} \mathrm{C}\right)$ after exposure of months [28]. It was also reported that Ringer's solution decreases the mechanical properties of Y-TZP upon prolonged exposures (months) [29]. By sharp contrast, Thompson and Rawlings [30] observed an immediate reduction of the flexural strength of Y-TZP of the order of $12-15 \%$, when tested in Ringer's solution. Such an immediate degradation may pose a severe limitation, to the point that those authors concluded that Y-TZP ceramics are simply unsuitable for biomedical applications. Moreover, when comparing cyclic crack velocities in partially stabilized tetragonal zirconia to stress-corrosion crack velocities measured under sustained loads, the cyclic crack velocities were found to be up to 7 times higher than the static ones. 
Correspondingly, threshold stress intensities were found to be almost $40 \%$ lower, than stresscorrosion crack velocities measured in identical environments under sustained-loading conditions [21]. Such observations may have serious implications on ceramic implants' mechanical long term reliability.

So far, it appears that a major effort has been invested in characterizing the extent of the tetragonal to monoclinic phase transformation, the circumstances leading to this formation, its mechanisms and influence on the fracture properties of the Y-TZP as a material per-se, emphasizing its beneficial effects on the fracture toughness and also its deleterious effects induced by swelling and surface microcracking. In parallel to this materials-science oriented body of research, little effort has been dedicated to the investigation of the structural/functional response of ceramic implants to repeated loading (fatigue). This characterization is of prime importance for the clinician and the patient alike (see e.g. [31]).

This paper reports the results of a systematic study of the fatigue performance of single-bodied Y-TZP zirconia dental implants, tested in room air and in $0.9 \%$ saline solution at room temperature. We apply the recently developed random spectrum testing approach [32] that allows to easily compare different test groups from a functional point of view that considers the structure, the geometry and the material altogether. Because of the extensive use of titanium in implant dentistry, the same study is conducted in parallel on geometrically identical CP-Ti implants in order to create a reference group.

\section{MATERIALS \& METHODS}

\subsection{Implants}

Two sets of geometrically identical monolithic implants (4.1 mm diameter, $20.5 \mathrm{~mm}$ length) were used for the study (Figure 1A). The tested non-commercial implants were made of partially stabilized zirconia (3Y-TZP, referred to as zirconia in the sequel), and commercially pure titanium (Grade $4 \mathrm{CP}$ ). No surface treatment was applied during manufacturing of the implants. The composition of the implants was supplied by the manufacturer and is detailed in Appendix 1.

\subsection{Implant mounting}

Steel sleeves were machined, and fitted to the implant diameter (Figure 1B). Each specimen was cemented to the sleeve with a high strength, 14 hour curing epoxy cement (Araldite 
standard $₫)$. Each sleeved specimen was mounted into a rigid steel block such as to expose its first thread (Figure 1C), and all were loaded from the same side (see the dot in Fig 1A and 1B).

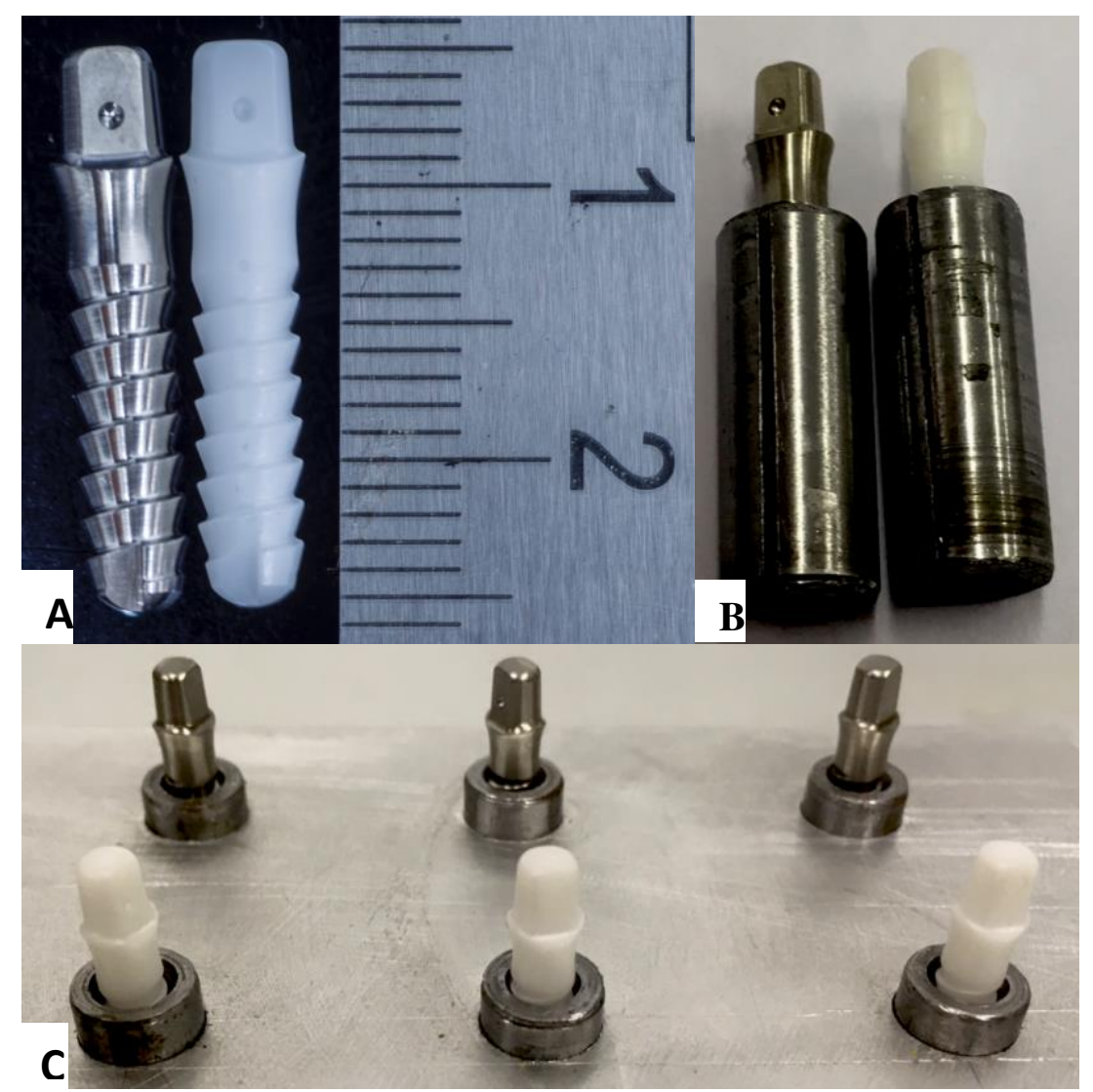

Figure 1: A: Zirconia (right) and CP-Ti (left) implants. Note the geometrical similarity. B: Implants mounted in the steel sleeve. C: Gluing setup showing the first exposed thread.

\subsection{Mechanical testing}

\section{Static}

The specimens were loaded in quasi-static compression $(0.5 \mathrm{~mm} / \mathrm{min}$ crosshead velocity) using a servo-hydraulic MTS machine. The specimens were mounted in the test rig at a $30^{\circ}$ angle with respect to the loading jig following the ISO standard 14801 standard [33]. Although this standard concerns titanium-made endosseous implants, we adopted this configuration to test the ceramic implants in order to evaluate as much as possible their functional performance. The very same mounting was used for both quasi-static and fatigue testing. Here, the specimens were bent until their fracture (clear load drop after linear load-displacement curve for the ceramic specimens) or noticeable plastic deformation (CP specimens). While in the first case, 
the peak load was recorded as an indication of the ceramic implant's strength, the "macroscopic yield point", corresponding to a deviation from linearity in the load displacement curve was recorded for the metallic implants, none of which actually fractured. Note that quasi-static testing was carried in room air and saline solution for the zirconia implants, and in room-air only for the CP-Ti as the latter is not susceptible to immediate significant corrosive attack, if at all. In all the tests, the implants were loaded using a flat-ended pushrod. All the fractures of the ceramic specimens occurred in the first thread (flush point corresponding to maximum bending moment) without any visible damage at the loading point, as an indication of the adequacy of this loading method (flat end pushrod). A total of 4 metallic implants were tested in air, 5 zirconia in air and 4 in saline solution.

\subsection{Random spectrum}

The random spectrum loading procedure was adopted as the optimal structural functionality test for the tested specific combination of implant geometry and material [32].

The idea of the test consists in subjecting the specimen to a random succession of loads, applied at various frequencies, whose amplitude varies between $0 \mathrm{~N}$ and a maximum value, selected according to the structural strength and working conditions. Likewise, the spectrum comprises random pauses during which the specimen is kept load-free, as occurs during oral function. The outcome of the test is the time to fracture of the specimen, so that when a group of specimens is tested, the outcome is a mean "longevity" value and its standard deviation $[32,34,35]$. It is important to note that all the test specimens undergo the very same spectrum loading, but each fails at a different time ("longevity"). The maximum test frequency was 3 $\mathrm{Hz}$, to somewhat mimic mastication. Testing was first carried out in room-air at ambient temperature, then in $0.9 \%$ saline solution in the subsequent step. Testing lasted until specimen fracture, or until a duration of 80,000 seconds without fracture, arbitrarily defined here as "runout" specimens based on the metallic test results.

A total of 15 metallic implants were tested in air, 15 zirconia in air and 15 more in saline solution. All the specimens were subjected to the same loading spectrum.

Since the random spectrum data reduction used in this work is not common and probably novel to some extent, a detailed account of its main steps is given next, point by point. 
1. Extract the data from the spectrum loading software main screen

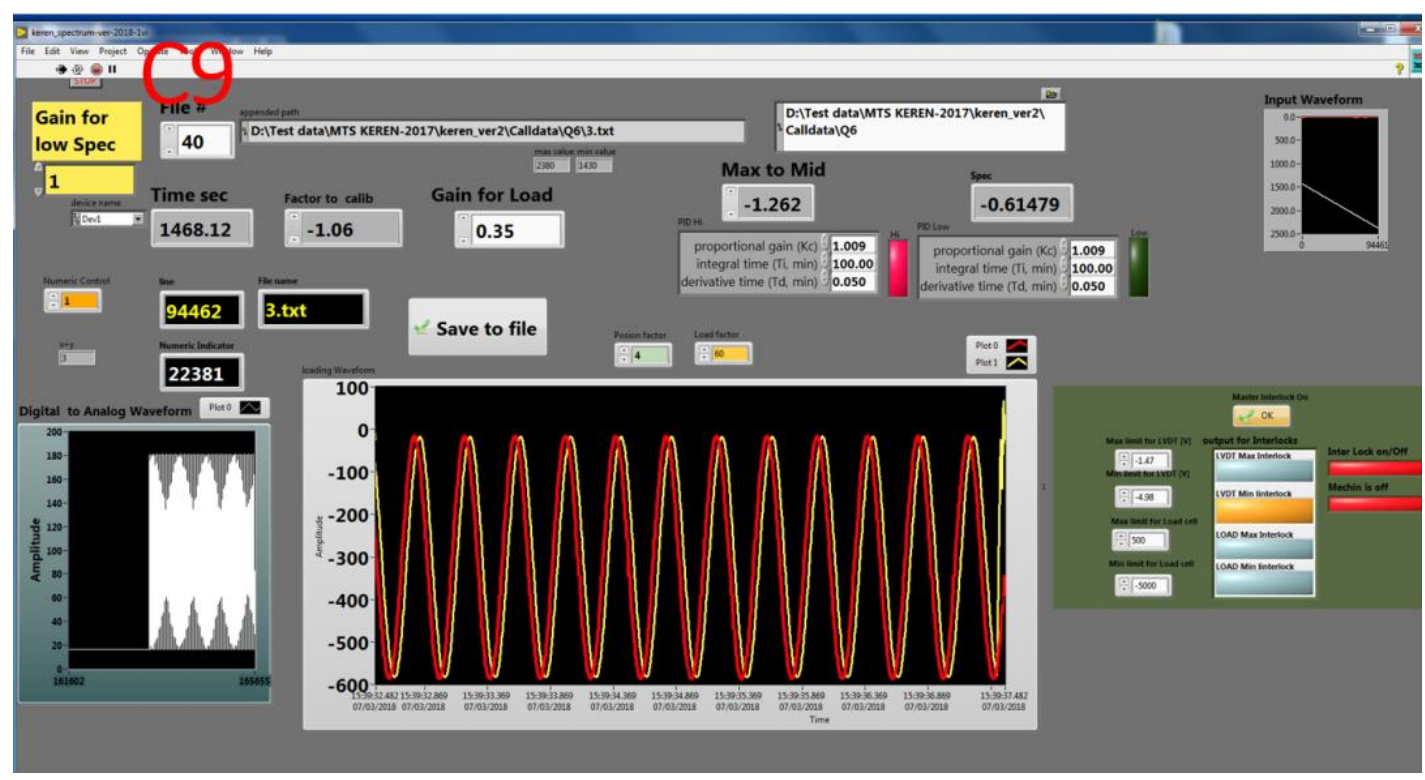

Figure 2: Screen capture of the test results. The red and yellow lines indicate the control/target and measured load signals, respectively.

The applied load is continuously monitored during the test and stored in buffered form. Fig. 2 shows a typical screen capture of the last recorded sequence at fracture. Given the length of the buffered signal, this sequence may or may not contain the starting point of the specific block. Therefore, the block number at which fracture occurred and the total elapsed time to fracture are recorded. In any case, both the prescribed and the recorded spectrum are available.

2. Data processing within the fracture block

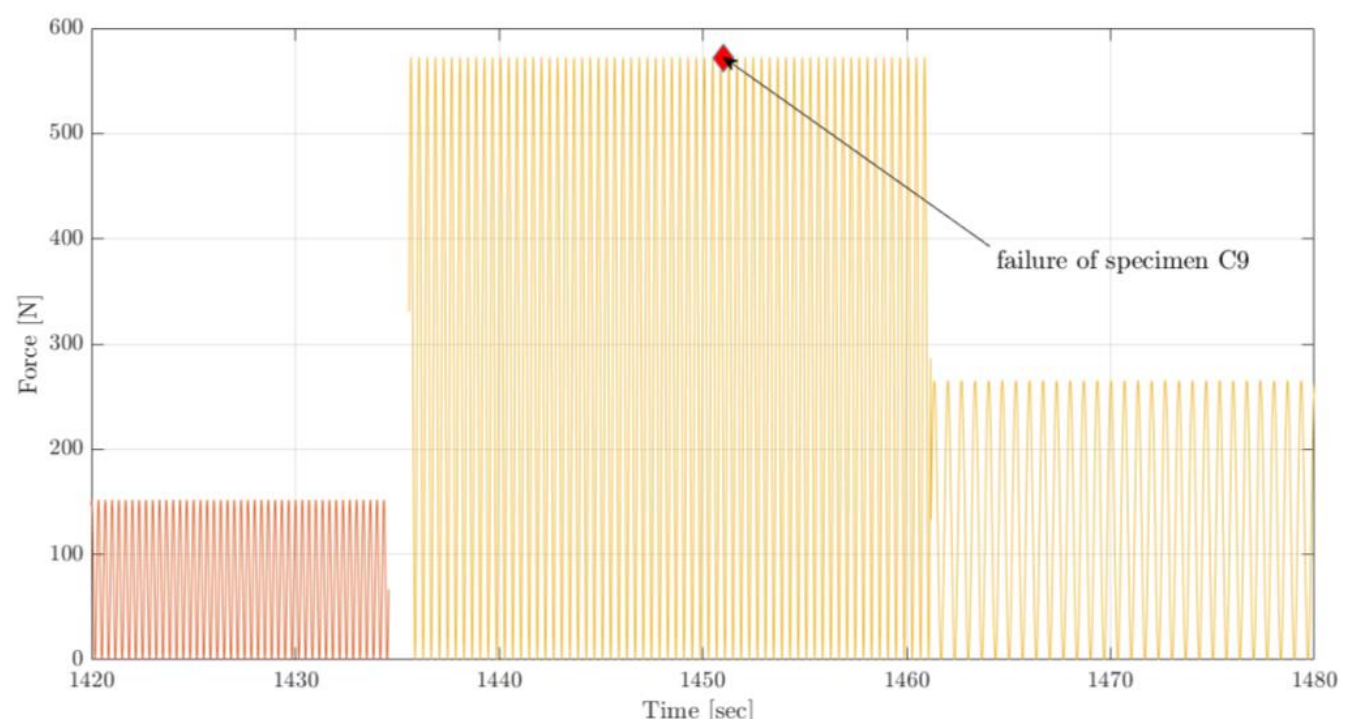


Figure 3: Fracture cycle of specimen C9

Fig. 3 illustrates the details of a load block and the point at which fracture occurred. This information, reveals the fracture load and the total fracture time, measured from the beginning of the test. The fracture time can later be converted into load cycles given the almost linear relationship between time and cycles (not shown here). If the origin of the block is recorded, as in Fig. 3, the exact total number of cycles to fracture is uniquely determined. If this is not the case, as in Fig 2 where the origin of the block is not recorded, the total number of cycles to failure will be bounded between a minimum (until the beginning of the block) and a maximum (end of the block) number of cycles, all determined from the prescribed spectrum.

The next step consists of counting the number of elapsed cycles for which the applied load exceeded or was equal to the recorded cyclic fracture load. If this number exceeds 1 , as for almost all specimens tested in this research, this is a clear indication of the operation of a cumulative damage mechanism, in this case fatigue.

\subsection{Statistical analysis}

For the various statistical analyses reported in the sequel, we used Wilcoxon Scores (Rank Sums) test without a-priori assumption of a statistical distribution.

\section{RESULTS}

\subsection{Quasi-static testing}

The quasi-static failure loads (fracture or yield) are summarized in Table 1. Additional details are provided in Table S1 (Supplementary Material section).

\begin{tabular}{|c|c|c|}
\hline Test group & \# specimens & $\begin{array}{c}\text { Fracture load } \\
\text { (average and standard } \\
\text { deviation) [N] }\end{array}$ \\
\hline Zirconia - room air & 5 & $802 \pm 107$ \\
\hline Zirconia - saline & 4 & $639 \pm 51$ \\
\hline CP Titanium - room air & 4 & $1242 \pm 85$ \\
\hline
\end{tabular}

Table 1: Quasi-static tests results 
For the zirconia implants, the median strength of the air group is $817 \mathrm{~N}$, while that of the saline group is $660.5 \mathrm{~N}$, which corresponds to a decrease in strength of $20 \%$. All the specimens fractured. Due to the limited sample size, statistical analysis of the results is precluded. The bending strength of the metallic samples is higher than that of the ceramic ones, noting that none of the CP-Ti implants actually fractured. With those figures in mind, we chose to perform the random spectrum testing with a peak load of $600 \mathrm{~N}$, as this load magnitude is relevant to oral mastication [35] and within the range of measured fracture loads (zirconia). It should be emphasized that the $600 \mathrm{~N}$ load is not the applied load in a classical cyclic loading test, but the maximum value that can be reached in the whole range of randomly determined load values of the spectrum.

\subsection{Random spectrum testing}

Table 2 summarizes the outcome of the random spectrum test results. None of the CP-Ti implants failed after completing 80,000 seconds of test (or more) in room-air. The detailed results are listed in Table S2 (Supplementary Material section).

\begin{tabular}{|c|c|c|}
\hline Test group & \# specimens & $\begin{array}{c}\text { Fracture time } \\
\text { (average and standard } \\
\text { deviation) [s] }\end{array}$ \\
\hline Zirconia - room air & 15 & $5131 \pm 5527$ \\
\hline Zirconia - saline & 15 & $2032 \pm 2912$ \\
\hline CP Titanium - room air & 15 & $>80,000$ (all runouts) \\
\hline
\end{tabular}

Table 2: Random spectrum test results.

The results of Table 2 show that on the average, the spectrum lifetime of zirconia implants tested in saline solution is shorter than that of implants tested in room air. In any case, none of the tested ceramic implants reached a longevity of 80,000 seconds like in the case of the titanium implants.

A statistically significant difference was found between the lifetime of zirconia tested in room air and in $0.9 \%$ saline solution $(\mathrm{p}=0.0144)$. Namely, the longevity of the room-air group was longer than that of the saline group (average time $5131 \pm 5527 \mathrm{~s}$, median 2738 s, vs. average time $2032 \pm 2912$, s and median 1449 s, respectively).

\subsection{Processing of the spectrum data}


From now on, we will consider cycles rather than time in order to pinpoint the potential operation of a fatigue (damage accumulation) failure mechanism, as opposed to monotonic overload once a critical value is first reached.

Table 3 concentrates the essential information obtained from the spectrum analysis, using the above-mentioned data-reduction procedure. The table includes the nominal time to failure and the fracture load $\left(\mathrm{Af}_{\mathrm{f}}\right.$, that was identified on the spectrum data on the last block to failure corresponding to the fracture time. Next, come the minimum and maximum number of cycles, for which the applied load (A) exceeded or equaled the fracture load, followed by the total number of cycles to failure.

As can be seen in Table 3, the minimum and maximum number of such cycles varies for each specimen. For almost all specimens, the number of such cycles is finite and vastly different from 1. In other words, each tested zirconia implant experienced a finite number of cycles during which the applied load amplitude, A, exceeded (or equaled) the recorded fracture load Af, so that failure due to monotonic overloading can be safely ruled out.

\begin{tabular}{|c|c|c|c|c|c|}
\hline $\begin{array}{c}\text { Specimen } \\
\text { number }\end{array}$ & $\begin{array}{c}\text { Nominal } \\
\text { time to } \\
\text { failure } \\
{[\mathbf{s}]}\end{array}$ & $\begin{array}{c}\text { Nominal } \\
\text { load at } \\
\text { fracture } \boldsymbol{A} \boldsymbol{f} \\
{[\mathbf{N}]}\end{array}$ & $\begin{array}{c}\text { Min total } \\
\text { cycles } \\
\mathbf{A} \geq \mathbf{A} \boldsymbol{f}\end{array}$ & $\begin{array}{c}\text { Max total } \\
\text { cycles } \\
\mathbf{A} \geq \mathbf{A} \boldsymbol{f}\end{array}$ & $\begin{array}{c}\text { Total } \\
\text { \# cycles }\end{array}$ \\
\hline $\mathrm{c} 9$ & 1468 & 572 & 13 & 63 & 2218 \\
\hline $\mathrm{c} 13$ & 2968 & 538 & 247 & 247 & 4046 \\
\hline $\mathrm{c} 14$ & 7497 & 547 & 362 & 457 & 9564 \\
\hline $\mathrm{c} 15$ & 17606 & 545 & 989 & 1036 & 22863 \\
\hline $\mathrm{c} 16$ & 17440 & 471 & 2961 & 2989 & 22786 \\
\hline $\mathrm{c} 17$ & 1450 & 572 & 11 & 11 & 2215 \\
\hline $\mathrm{c} 19$ & 9527 & 530 & 111 & 163 & 12358 \\
\hline $\mathrm{c} 20$ & 1451 & 572 & 11 & 11 & 2218 \\
\hline $\mathrm{c} 21$ & 2237 & 543 & 245 & 300 & 3194 \\
\hline $\mathrm{c} 22$ & 2741 & 575 & 13 & 108 & 3822 \\
\hline $\mathrm{c} 23$ & 2738 & 575 & 13 & 108 & 3812 \\
\hline $\mathrm{c} 24$ & 1452 & 572 & 13 & 63 & 2218 \\
\hline $\mathrm{c} 25$ & 3436 & 552 & 178 & 178 & 4674 \\
\hline $\mathrm{c} 26$ & 2224 & 532 & 101 & 155 & 3192 \\
\hline $\mathrm{c} 27$ & 2736 & 575 & 7 & 108 & 3809 \\
\hline
\end{tabular}

\begin{tabular}{|c|c|c|c|c|c|}
\hline $\begin{array}{c}\text { Specimen } \\
\text { number }\end{array}$ & $\begin{array}{c}\text { Nominal } \\
\text { time to } \\
\text { failure } \\
{[\mathbf{s}]}\end{array}$ & $\begin{array}{c}\text { Nominal } \\
\text { load at } \\
\text { fracture } \boldsymbol{A} \boldsymbol{f} \\
{[\mathbf{N}]}\end{array}$ & $\begin{array}{c}\text { Min total } \\
\text { cycles } \\
\mathbf{A} \geq \mathbf{A} \boldsymbol{f}\end{array}$ & $\begin{array}{c}\text { Max total } \\
\text { cycles } \\
\mathbf{A} \geq \mathbf{A} \boldsymbol{f}\end{array}$ & $\begin{array}{c}\text { Total } \\
\text { \# cycles }\end{array}$ \\
\hline $\mathrm{c} 39$ & 1449 & 573 & 13 & 64 & 2210 \\
\hline $\mathrm{c} 40$ & 27 & 350 & 1 & 1 & 1 \\
\hline $\mathrm{c} 41$ & 1446 & 573 & 3 & 3 & 2205 \\
\hline $\mathrm{c} 42$ & 1463 & 573 & 13 & 64 & 2247 \\
\hline
\end{tabular}




\begin{tabular}{|c|c|c|c|c|c|}
\hline $\mathrm{c} 43$ & 1458 & 573 & 13 & 64 & 2235 \\
\hline $\mathrm{c} 44$ & 142 & 417 & 33 & 61 & 125 \\
\hline $\mathrm{c} 45$ & 2975 & 538 & 252 & 275 & 4070 \\
\hline $\mathrm{c} 46$ & 2746 & 575 & 145 & 239 & 3832 \\
\hline $\mathrm{c} 47$ & 2118 & 484 & 512 & 569 & 3037 \\
\hline $\mathrm{c} 48$ & 316 & 484 & 5 & 35 & 384 \\
\hline $\mathrm{c} 49$ & 2743 & 575 & 13 & 108 & 3824 \\
\hline $\mathrm{c} 50$ & 508 & 451 & 41 & 41 & 689 \\
\hline $\mathrm{c} 51$ & 11933 & 553 & 517 & 532 & 15714 \\
\hline $\mathrm{c} 52$ & 142 & 417 & 32 & 61 & 128 \\
\hline $\mathrm{c} 54$ & 1018 & 510 & 5 & 5 & 1518 \\
\hline
\end{tabular}

Table 3: Data processed from the random spectrum tests of zirconia implants. The nominal time and fracture loads are listed in columns 2 and 3. Columns 4 and 5 list the minimum and maximum number of cycles for which the experienced load exceeded the measured fracture load (see explanation in Appendix 4). The rightmost column lists the values of the fracture load.

Figure 4 shows the total number of cycles to failure for each tested group (Table 3 ). This figure reflects the earlier statistical observation that the fatigue longevity of the tested implants is significantly higher in room air than in the saline solution.



Figure 4: Total number of cycles to failure for the two groups of implants. Note that overall, the implants tested in the saline solution underwent a smaller number of cycles, as indicated by the earlier statistical analysis of the results.

Figure 5 shows the number of specimens for binned fracture loads, up to monotonic fracture, represented by one specimen at this value. The same histograms show the peak load value used in those tests $(600 \mathrm{~N})$, as well as the average monotonic fracture load of each group according to the test atmosphere. 
The median fatigue fracture load of the zirconia implants is $552 \mathrm{~N}$ in room air vs. 538 in $0.9 \%$ saline solution group. Despite some apparent shift in the results, the statistical analysis revealed no real difference between the spectrum failure load magnitude recorded in different test environments ( $\mathrm{p}=0.3484)$. This observation allows us to lump the two groups of specimens into a single group, in terms of fracture load.



Figure 5: Histogram showing the number of zirconia specimens vs. their spectrum fracture load.

Figure 6 shows the number of cycles for which the load was superior or equal to the recorded fracture load. Note that this number is almost always superior to 1 , both in room air and in $0.9 \%$ saline solution indicating unambiguously the operation of a fatigue failure mechanism.

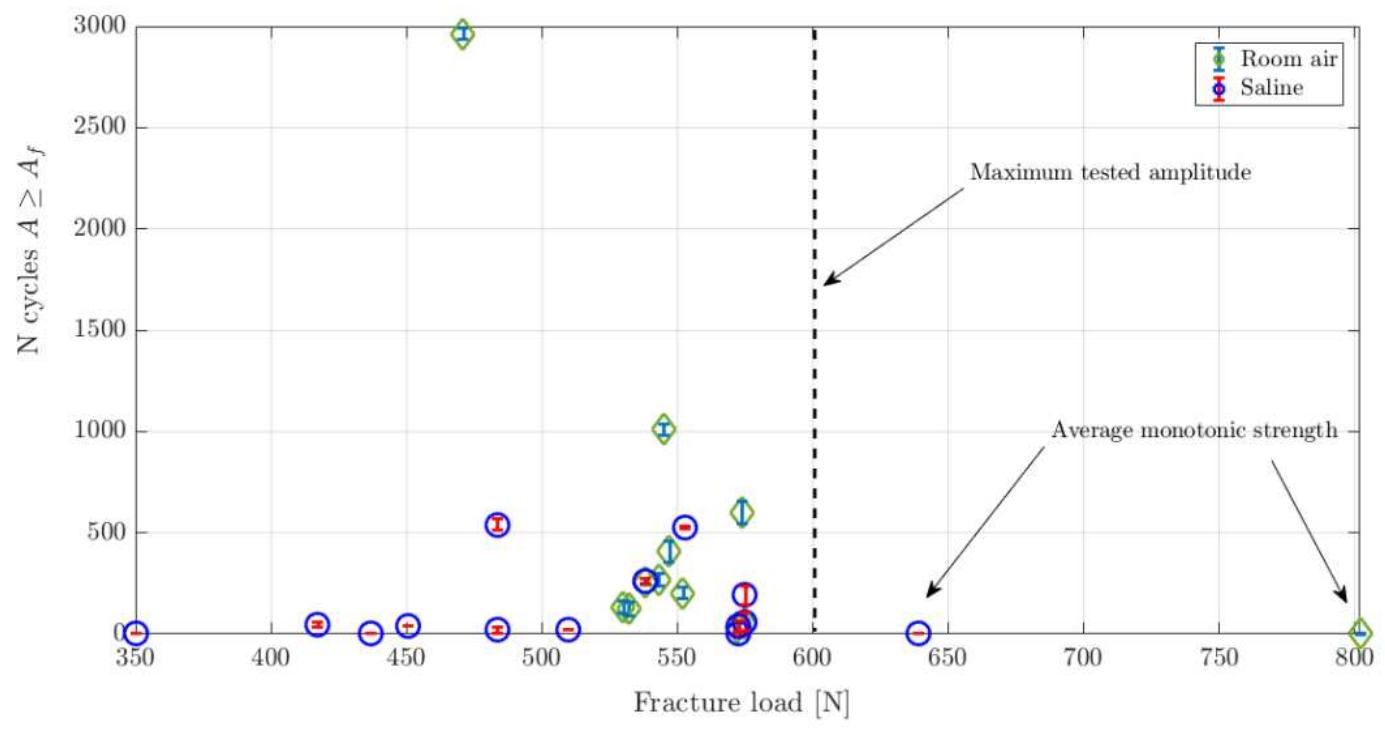


Figure 6: Number of cycles for which the load was superior or equal to the recorded fracture load. Note that this number is almost always superior to 1 , indicating a fatigue failure mechanism. The average monotonic fracture load is added, for which the corresponding number of cycles is 1 .

Further insight can be gained by considering the ratio of the number of cycles exceeding or equal to the fracture strength to the total number of cycles for each specimen. This ratio is plotted as a function of the fatigue fracture strength of all the tested zirconia specimens, lumped together, in Figure 7.

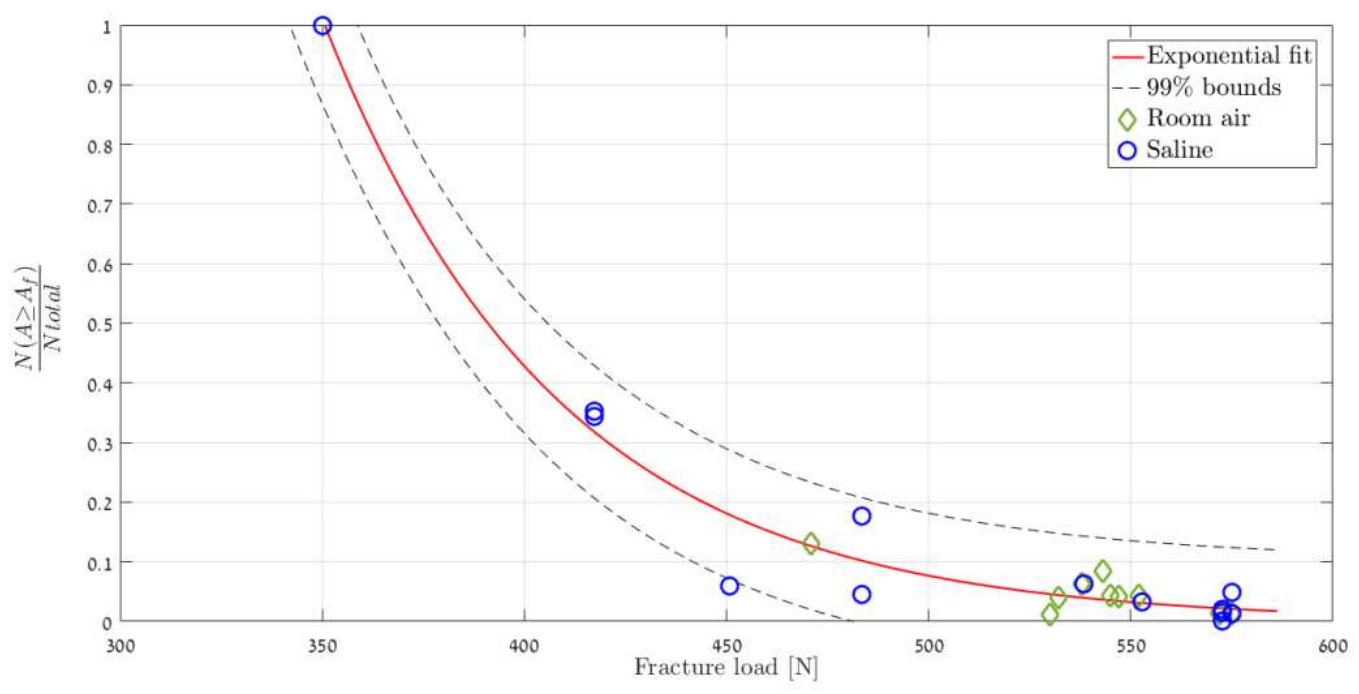

Figure 7: Ratio of the average number of cycles for which the amplitude is equal or exceeds the fracture load to the total number of cycles (Table 3 ) as a function of the fracture load. 99\% estimated bounds are plotted.

Figure 7 shows that the relationship between the two variables is exponential. As the fracture load increases, the percentage of relative cycles decreases.

\section{DISCUSSION}

This study is the first of its kind in which implants made of zirconia are systematically compared for their fatigue response from a functional standpoint, using the random spectrum approach. This technique has been repeatedly suggested, e.g. by $[19,20]$, even if the goal was primarily to investigate cyclic crack growth and retardation/acceleration effects. Here, each experimental group is subjected to the very same spectrum, so that one average longevity and its standard deviation define the group unambiguously. 
It must be realized that the maximum load that can occur during a cycle, $600 \mathrm{~N}$, is roughly 0.75 of that of the (dry) zirconia and half the strength of the CP-Ti. Although high, such a load value can be encountered during mastication, as the load varies with the individual, implant location and parafunctional habits [35]. As such, there is a wide difference in terms of relative loads applied to each group of materials, but this is nevertheless the best way to compare them vs. loads that could actually be experienced during mastication, irrespective of the implant's material.

Without further consideration, it is clear that the CP-Ti implants offer a clear advantage in terms of fatigue longevity since none off those implants fractured in both test atmospheres after 80,000 seconds and more. As such, this group will only be considered as a reference group without further comparison with the ceramic implants.

Although the present tests are based on random and not cyclic loading, one can observe in Figure 5 a clustering of the failure loads, in both room air and saline solution, between 525$575 \mathrm{~N}$, irrespective of the test group, as shown by the statistical analysis.

Assuming a representative wet fracture strength of $640 \mathrm{~N}, 500 \mathrm{~N}-$ a lower bound for 525-575 $\mathrm{N}$ - represents $0.78(0.8)$ of that value. But mainly, those results imply that testing should be carried out in saline solution, or any solution that is representative of the intraoral atmosphere [36], in order to obtain conservative estimates.

While it is observed that the saline solution causes a marked decrease in the implant fatigue longevity and most likely its quasi-static strength in accord with [30], it does not influence the fatigue fracture load, which might indicate the implant's environment has an effect on the rate of cyclic damage and accumulation. However, complementary fracture mechanics studies are needed to pinpoint separately crack initiation and growth in such materials as a function of the test atmosphere

The results of this study suggest that in order to assess a "safe load" value for zirconia dental implants, the latter should be tested first quasi-statically e.g. in saline solution and the safe maximum load would then be of the order of 0.8 times the wet fracture strength, that in itself is inferior to the dry one. Such a design guideline for random spectrum testing has not been proposed before, as done here on the basis of experimental testing.

In that context, an additional interesting outcome of this work can be found in Figure 7. This figure shows that the average relative number of cycles (exceeding fracture load over total number of cycles) is exponentially related to the spectrum fracture load. In other words, a "weak" (low Af)" implant will have endured more "strong" (A> Af) cycles than a stronger one, that can only withstand a (very) few strong (A> Af) cycles. Stated otherwise, the spectrum fracture load is correlated to the both number and the nature of the cycles that preceded fracture. 
Such observation might imply that with time and mild usage, the anticipated fracture load will decrease as a result of damage accumulation, thereby excluding additional potential high amplitude (A> Af) load excursions. Taking 0.8 times the wet static fracture strength as a reference load, such load value will not allow for higher load excursions, as in the case of parafunctional habits.

Beyond the "design guidelines" that come from this work, the present research confirms the operation of a fatigue failure mechanism in ceramic dental implants. The very observation that a specimen could withstand tens to hundreds of cycles exceeding its (fatigue) fracture load shows without any ambiguity that this specimen has experienced fatigue damage. Unlike the case of most metallic materials, the identification of a fatigue fracture mechanism in such ceramics is complicated. A detailed fractographic characterization can be found in [37], in which the relative occurrence of cleavage vs. intergranular fracture is correlated with the nature of the failure mechanism and testing medium. Cyclic plasticity is irrelevant in this case so that another type of damage accumulation should be identified [38]. This was not the goal of the present work, but future studies that would look carefully at the tetragonal to monoclinic transformation under cyclic loading and its effect on the surface of the specimen (e.g. swelling or microcracking) will no doubt shed additional light on this failure mechanism. In parallel, one can safely propose that the presence of a liquid atmosphere decreases the surface energy of the ceramic, just like it does for metals, so that the resulting fracture toughness decreases and the propensity to crack nucleation, and perhaps propagation, increases accordingly (see also [24]).

As a final remark, this work illustrates the feasibility of an alternative fatigue testing procedure to the characterization of ceramic dental implants, emphasizing their functionality, therefore unraveling a potential for future comparative and quantitative studies. As such, this study expands the range of applications, previously restricted to metallic implants. This work shows that the random spectrum methodology has a wide range of potential applications for ceramic implants too, regarding the grade of the material, the nature of the surface treatments and the overall mechanical design among other parameters. Yet, it should be kept in mind that the actual figures reported in this work were obtained for a specific kind of zirconia implants and therefore cannot be generalized to all kinds of ceramic implants.

\section{CONCLUSIONS}

This study shows that, for the range of loads considered in this study, Y-TZP implants are less resistant to fatigue than their Ti-based counterpart. 
Y-TZP implants are clearly shown to fail by a fatigue mechanism under random spectrum loading.

Saline solution seems to reduce the monotonic fracture strength of Y-TZP implants by some $20 \%$, and it also reduces their fatigue longevity significantly without affecting their spectrum fatigue fracture strength.

To design Y-TZP implants, one should first consider their monotonic fracture strength in saline (or other intraoral) representative solution.

For the kind of implants tested in this work, one should make sure that 0.8 of this wet strength will not be exceeded during the life service of the implant.

\section{ACKNOWLEDGEMENT}

Mr. Amir Reuven is kindly acknowledged for his technical assistance with the tests, and Mr. Sagi Chen for his assistance with the random spectrum code.

This research did not receive any specific grant from funding agencies in the public, commercial, or not-for-profit sectors. 
APPENDIX 1: Composition of the tested implants

(provided by the manufacturer, spectrometric analysis)

CP-Titanium, Grade 4

\begin{tabular}{|c|c|c|c|c|c|c|c|c|c|}
\hline Element & $\mathrm{H}_{2}$ & $\mathrm{~N}_{2}$ & $\mathrm{O}_{2}$ & $\mathrm{Fe}$ & $\mathrm{C}$ & $\mathrm{B}$ & $\mathrm{Si}$ & $\mathrm{Y}$ & $\mathrm{Ti}$ \\
\hline wt \% & 0.0003 & 0.002 & 0.32 & 0.2 & 0.002 & $<0.003$ & $<0.03$ & $<0.005$ & Bal. \\
\hline
\end{tabular}

Zirconia

\begin{tabular}{|c|c|c|c|c|c|}
\hline Element & $\begin{array}{c}\mathrm{ZrO}_{2}+ \\
\mathrm{HfO}_{2}+\mathrm{Y}_{2} \mathrm{O}_{3}\end{array}$ & $\mathrm{Y}_{2} \mathrm{O}_{3}$ & $\mathrm{HfO}_{2}$ & $\mathrm{Al}_{2} \mathrm{O}_{3}$ & $\begin{array}{c}\text { Other oxides } \\
\text { Total }\end{array}$ \\
\hline wt \% & 99.35 & 5.73 & 1.46 & 0.33 & 0.32 \\
\hline
\end{tabular}




\section{Declarations of interest}

None.

Data availability

The raw/processed data are available upon written request from the authors. 


\section{REFERENCES}

[1] S.T. Becker, B.E. Beck-Broichsitter, C.M. Rossmann, E. Behrens, A. Jochens, J. Wiltfang, Long-term Survival of Straumann Dental Implants with TPS Surfaces: A Retrospective Study with a Follow-up of 12 to 23 Years, Clin. Implant Dent. Relat. Res. 18 (2016) 480-488. doi:10.1111/cid.12334.

[2] T. Horikawa, T. Odatsu, T. Itoh, Y. Soejima, H. Morinaga, N. Abe, N. Tsuchiya, T. Iijima, T. Sawase, Retrospective cohort study of rough-surface titanium implants with at least 25 years' function., Int. J. Implant Dent. 3 (2017) 42. doi:10.1186/s40729-0170101-7.

[3] V. Chappuis, R. Buser, U. Brägger, M.M. Bornstein, G.E. Salvi, D. Buser, Long-Term Outcomes of Dental Implants with a Titanium Plasma-Sprayed Surface: A 20-Year Prospective Case Series Study in Partially Edentulous Patients, Clin. Implant Dent. Relat. Res. 15 (2013) 780-790. doi:10.1111/cid.12056.

[4] J. Chevalier, L. Gremillard, Ceramics for medical applications: A picture for the next 20 years, J. Eur. Ceram. Soc. 29 (2009) 1245-1255.

doi:10.1016/j.jeurceramsoc.2008.08.025.

[5] Y. Zhang, I. Sailer, B.R. Lawn, Fatigue of dental ceramics, J. Dent. 41 (2013) 11351147. doi:10.1016/j.jdent.2013.10.007.

[6] K.T. Kim, M.Y. Eo, T.T.H. Nguyen, S.M. Kim, General review of titanium toxicity, Int. J. Implant Dent. 5 (2019) 10. doi:10.1186/s40729-019-0162-x.

[7] B.R. Chrcanovic, J. Kisch, T. Albrektsson, A. Wennerberg, Bruxism and dental implant treatment complications: A retrospective comparative study of 98 bruxer patients and a matched group, Clin. Oral Implants Res. (2016) 1-9. doi:10.1111/clr.12844.

[8] S. Pieralli, R.-J. Kohal, E. Lopez Hernandez, S. Doerken, B.C. Spies, Osseointegration of zirconia dental implants in animal investigations: A systematic review and metaanalysis., Dent. Mater. 34 (2018) 171-182. doi:10.1016/j.dental.2017.10.008.

[9] C. Oblak, A. Kocjan, P. Jevnikar, T. Kosmac, The effect of mechanical fatigue and accelerated ageing on fracture resistance of glazed monolithic zirconia dental bridges, J. Eur. Ceram. Soc. 37 (2017) 4415-4422. doi:10.1016/j.jeurceramsoc.2017.04.048.

[10] C. Sanon, J. Chevalier, T. Douillard, M. Cattani-Lorente, S.S. Scherrer, L. Gremillard, A new testing protocol for zirconia dental implants, Dent. Mater. 31 (2015) 15-25. doi:10.1016/j.dental.2014.09.002.

[11] J.R. Kelly, I. Denry, Stabilized zirconia as a structural ceramic: An overview, Dent. Mater. 24 (2008) 289-298. doi:10.1016/j.dental.2007.05.005.

[12] J. Chevalier, C. Olagnon, G. Fantozzi, B. Galès, Crack Propagation Behavior of YTZP Ceramics, J. Am. Ceram. Soc. 78 (1995) 1889-1894. doi:10.1111/j.11512916.1995.tb08905.x.

[13] D. Hashim, N. Cionca, D.S. Courvoisier, A. Mombelli, A systematic review of the clinical survival of zirconia implants, Clin. Oral Investig. 20 (2016) 1403-1417. doi:10.1007/s00784-016-1853-9.

[14] S. Roehling, H. Woelfler, S. Hicklin, H. Kniha, M. Gahlert, A Retrospective Clinical Study with Regard to Survival and Success Rates of Zirconia Implants up to and after 7 Years of Loading, Clin. Implant Dent. Relat. Res. 18 (2016) 545-558. doi:10.1111/cid.12323.

[15] B. Pjetursson, A. Asgeirsson, M. Zwahlen, I. Sailer, Improvements in implant dentistry over the last decade: comparison of survival and complication rates in older and newer 
publications, Int. J. Oral Maxillofac. Implants. 29 (2014) 308-24.

[16] B.C. Spies, J. Nold, K. Vach, R.J. Kohal, Two-piece zirconia oral implants withstand masticatory loads: An investigation in the artificial mouth, J. Mech. Behav. Biomed. Mater. 53 (2016) 1-10. doi:10.1016/j.jmbbm.2015.07.005.

[17] R.J. Kohal, M. Wolkewitz, A. Tsakona, The effects of cyclic loading and preparation on the fracture strength of zirconium-dioxide implants: An in vitro investigation, Clin. Oral Implants Res. 22 (2011) 808-814. doi:10.1111/j.1600-0501.2010.02067.x.

[18] K. Shemtov-Yona, D. Rittel, L. Levin, E.E. Machtei, The effect of oral-like environment on dental implants' fatigue performance, Clin. Oral Implants Res. 25 (2014) 1-5. doi:10.1111/clr.12084.

[19] R. Dauskardt, W. Yu, R. Ritchie, Fatigue Crack Propagation in TransformationToughened Zirconia Ceramic, J. Am. Ceram. Soc. 70 (1987) 248-252. doi:10.1111/j.1151-2916.1987.tb04889.x.

[20] R.H. Dauskardt, W.C. Carter, D.K. Veirs, R.O. Ritchie, Transient subcritical crackgrowth behavior in transformation-toughened ceramics, Acta Metall. Mater. 38 (1990) 2327-2336. doi:10.1016/0956-7151(90)90099-3.

[21] R.H. Dauskardt, D.B. Marshall, R.O. Ritchie, Cyclic Fatigue-Crack Propagation in Magnesia-Partially-Stabilized Zirconia Ceramics, J. Am. Ceram. Soc. 73 (1990) 893903. doi:10.1111/j.1151-2916.1990.tb05132.x.

[22] R.O. Ritchie, Fatigue of Brittle Materials, Compr. Struct. Integr. 4 (2003) 359-388. doi:10.1361/asmhba0002417.

[23] S. Suresh, Fatigue of materials, Cambridge University Press, 1998.

[24] C. Olagnon, J. Chevalier, V. Pauchard, Global description of crack propagation in ceramics, J. Eur. Ceram. Soc. 26 (2006) 3051-3059. doi:10.1016/j.jeurceramsoc.2005.11.004.

[25] L.A. Sylva, S. Suresh, Crack growth in transforming ceramics under cyclic tensile loads, J. Mater. Sci. 24 (1989) 1729-1738. doi:10.1007/BF01105698.

[26] M. Yoshimura, T. Noma, K. Kawabata, S. Somiya, Role of H20 on the degradation process of Y-TZP, J. Mater. Sci. Lett. 6 (1987) 465-7.

[27] M. Yoshimura, Phase stability of zirconia, Ceram. Bull. 67 (1988) 1950-1955.

[28] M. Cattani-Lorente, S.S. Scherrer, P. Ammann, M. Jobin, H.W.A. Wiskott, Low temperature degradation of a Y-TZP dental ceramic, Acta Biomater. 7 (2011) 858865. doi:10.1016/j.actbio.2010.09.020.

[29] J.L. Drummond, In vitro aging of Yttria-stabilized zirconia, J. Am. Ceram. Soc. 76 (1989) 675-676.

[30] I. Thompson, R.D. Rawlings, Mechanical behaviour of zirconia and zirconiatoughened alumina in a simulated body environment, Biomaterials. 11 (1990) 505508. doi:10.1016/0142-9612(90)90066-Y.

[31] C. Sanon, J. Chevalier, T. Douillard, R.J. Kohal, P.G. Coelho, J. Hjerppe, N.R.F.A. Silva, Low temperature degradation and reliability of one-piece ceramic oral implants with a porous surface, Dent. Mater. 29 (2013) 389-397. doi:10.1016/j.dental.2013.01.007.

[32] K. Shemtov-Yona, D. Rittel, Random spectrum loading of dental implants: An alternative approach to functional performance assessment, J. Mech. Behav. Biomed. Mater. 62 (2016) 1-9. doi:10.1016/j.jmbbm.2016.04.030.

[33] ISO, ISO 14801:2016(en), Dentistry — Implants — Dynamic loading test for endosseous dental implants, 2016. https://www.iso.org/obp/ui/\#iso:std:iso:14801:ed3:v1:en (accessed February 10, 2017).

[34] K. Shemtov-Yona, D. Rittel, Fatigue failure of dental implants in simulated intraoral media, J. Mech. Behav. Biomed. Mater. 62 (2016) 636-644. doi:10.1016/j.jmbbm.2016.05.028.

[35] K. Shemtov-Yona, D. Rittel, Fatigue of Dental Implants: Facts and Fallacies, Dent. J. 
4 (2016) 16. doi:10.3390/dj4020016.

[36] A.E. Medvedev, R. Lapovok, Y. Estrin, T.C. Lowe, V.N. Anumalasetty, Bending Fatigue Testing of Commercial Purity Titanium for Dental Implants, Adv. Eng. Mater. 18 (2016) 1166-1173. doi:10.1002/adem.201600002.

[37] K. Shemtov-Yona, M. Özcan, D. Rittel, Fractographic characterization of fatigued zirconia dental implants tested in room air and saline solution, Eng. Fail. Anal. (2019). doi:10.1016/j.engfailanal.2018.10.020.

[38] A.G. Evans, Fatigue in ceramics, Int. J. Fract. 16 (1980) 485-498. doi:10.1007/BF02265214. 